\title{
Coarse-grained molecular models for high-throughput and multi-scale functional investigations
}

\section{Kay Hamacher}

Address: Bioinformatics \& Theo. Biology Group, Department of Biology, Technische Universität Darmstadt, Schnittspahnstr. 10,64287 Darmstadt, Germany

Email: Kay Hamacher - hamacher@bio.tu-darmstadt.de

from 3rd German Conference on Chemoinformatics

Goslar, Germany. II-I3 November 2007

Published: 26 March 2008

Chemistry Central Journal 2008, 2(SuppI I):SI4 doi:I0.II86/I752-I53X-2-SI-SI4

This abstract is available from: http://www.journal.chemistrycentral.com/content/2/SI/SI4

(C) 2008 Hamacher

In this contribution we discuss the development, implementation, and the subsequent application of in silico, coarse-grained molecular models. The proposed approach allows for bridging a methodological gap between sequence-based bioinformatics and molecular dynamics simulations: molecular interactions can be modeled based on physio-chemical model in a highly parallelizable and high-throughput-ready fashion.

We demonstrate the broad applicability of such an approach by discussing the assembly of the bacterial ribosome [1] and potential points of interference by antibiotica, HIV protease [2] and its evolutionary dynamics en route to drug resistance [3]. These methodologies were further applied to a signaling cascade in leukemia $[4,5]$ and can be used for design by e.g. global optimization techniques [6]. In addition we show recent results on further computational improvements and additional observations from a physicist's/chemist's point of view.

\section{References}

I. Hamacher K, Trylska J, McCammon JA: Dependency Map of Proteins in the Small Ribosomal Subunit. PLoS Comput Biol 2006, 2:el0.

2. Hamacher K, McCammon JA: Computing the Amino Acid Specificity of Fluctuations in Biomolecular Systems. J Chem Theo Comp 2006, 2:873.

3. Hamacher K: Gene 2007. submitted

4. Hamacher K, Hübsch A, McCammon JA: A minimal model for stabilization of biomolecules by hydrocarbon cross-linking. I Chem Phys 2006, I 24:164907-1649I4.

5. Hamacher K: Information Theoretical Measures to Analyze Trajectories in Rational Molecular Design. J Comp Chem 2007, 28:2576-2580.
6. Hamacher K: Adaptation in Stochastic Tunneling Global Optimization of Complex Potential Energy Landscapes. Europhys Lett 2006, 74:944-950.

7. Hamacher K: Energy landscape paving as a perfect optimization approach under detrended fluctuation analysis. Physica $\mathrm{A}$ 2007, 378:307-3I4. 